\title{
Worsening renal function in patients hospitalised for acute heart failure: Clinical implications and prognostic significance
}

\author{
Marco Metra ${ }^{\mathrm{a}, *}$, Savina Nodari ${ }^{\mathrm{a}}$, Giovanni Parrinello ${ }^{\mathrm{b}}$, Tania Bordonali ${ }^{\mathrm{a}}$, Silvia Bugatti ${ }^{\mathrm{a}}$, \\ Rossella Danesi $^{a}$, Benedetta Fontanella ${ }^{a}$, Carlo Lombardi ${ }^{\text {a }}$, Patrizia Milani ${ }^{\mathrm{a}}$, Giulia Verzura ${ }^{\mathrm{a}}$, \\ Gadi Cotter $^{\mathrm{c}}$, Howard Dittrich ${ }^{\mathrm{d}}$, Barry M. Massie ${ }^{\mathrm{e}}$, Livio Dei Cas ${ }^{\mathrm{a}}$ \\ ${ }^{a}$ Section on Cardiovascular Diseases, Department of Experimental and Applied Medicine, University of Brescia, Italy \\ ${ }^{\mathrm{b}}$ Section of Medical Statistics, Department of Medical Sciences and Biotechnology, University of Brescia, Italy \\ ${ }^{\mathrm{c}}$ Momentum-Research, Durham, NC, USA \\ d NovaCardia Inc., San Diego, Ca, USA \\ ${ }^{\mathrm{e}}$ Cardiology Division, University of California and the Department of Veterans Affairs Medical Center, San Francisco, Ca, USA
}

Received 29 September 2007; received in revised form 12 January 2008; accepted 21 January 2008

\begin{abstract}
Background: Renal function is a powerful prognostic variable in patients with heart failure (HF). Hospitalisations for acute HF (AHF) may be associated with further worsening of renal function (WRF).

Methods and results: We analysed the clinical significance of WRF in 318 consecutive patients admitted at our institute for AHF. WRF was defined as the occurrence, at any time during the hospitalisation, of both a $\geq 25 \%$ and a $\geq 0.3 \mathrm{mg} / \mathrm{dL}$ increase in serum creatinine (s-Cr) from admission (WRF-Abs-\%).

Results: Patients were followed for $480 \pm 363$ days. Fifty-three patients (17\%) died and 132 (41\%) were rehospitalised for HF. WRF-Abs- $\%$ occurred in 107 (34\%) patients. At multivariable survival analysis, WRF-Abs-\% was an independent predictor of death or HF rehospitalisation (adjusted HR, 1.47; 95\%CI, 1.13-1.81; $p=0.024$ ). The independent predictors of WRF-Abs-\%, evaluated using multivariable logistic regression, were history of chronic kidney disease $(p=0.002)$, LV ejection fraction $(p=0.012)$, furosemide daily dose $(p=0.03)$ and NYHA class $(p=0.05)$ on admission.

Conclusion: WRF is a frequent finding in patients hospitalised for AHF and is associated with a poor prognosis. Severity of HF and daily furosemide dose are the most important predictors of the occurrence of WRF.

(C) 2008 Published by Elsevier B.V. on behalf of European Society of Cardiology.
\end{abstract}

Keywords: Acute heart failure; Renal function; Prognosis

\section{Introduction}

Renal dysfunction is a frequent finding in patients with heart failure (HF) and is a powerful independent prognostic factor for adverse outcomes [1-8]. Its prevalence increases

\footnotetext{
* Corresponding author. Cattedra di Cardiologia, c/o Spedali Civili, Piazza Spedali Civili, 25100 Brescia, Italy. Tel.: +39030 3995573; fax: +39030 3700359.

E-mail address: metramarco@libero.it (M. Metra).
}

in patients with more severe HF. More than half of the patients hospitalised for HF have some degree of impairment of renal function, and moderate to severe impairment has been reported in $30-35 \%$ of cases [7,9-13]. Hospitalisation for acute $\mathrm{HF}$ is associated with further worsening of renal function (WRF) in $30-50 \%$ of patients, depending on the specific definition utilized, and this is associated with prolonged length of hospital stay, increased healthcare costs, increased in-hospital mortality, and higher rates of rehospitalisation and death post-discharge [9-11,14-17]. 
However, these data are derived primarily from retrospective analyses of patients' records from databases of multiple centers and are often limited to patients $\geq 65$ years of age $[9-11,13,15]$. With two exceptions $[16,17]$, data on treatment, an important determinant of renal function changes, were not available or utilized. The studies also had relatively short follow-up duration, limited to the inhospital course in many studies $[13,15,16]$ and up to 6 months in others $[9,10,17]$. Lastly, there is no consensus on how best to define WRF, with some studies utilizing the absolute [9$13,15-17]$ and others using relative $[14,18]$ changes in serum creatinine (s-Cr) values.

The aim of the present study was to assess the incidence of WRF, defined as an absolute or relative increase in s-Cr, as well as its one-year prognostic value, clinical characteristics and risk factors in a consecutive series of patients admitted for acute HF.

\section{Methods}

\subsection{Patients}

We prospectively enrolled all qualifying patients admitted for acute HF at our Institute of Cardiology in Brescia, Italy, from June 2003 to June 2006. To be included into the study patients had to satisfy the diagnostic criteria of acute HF, as established by the European Society of Cardiology guidelines [19], and require treatment with an intravenous agent, which in all cases included furosemide with or without other vasoactive medications. All patients gave their informed written consent to the protocol, which was approved by the local Ethics Committee. We excluded patients unable to give informed consent and those with evidence of acute coronary syndrome, acute arrhythmia, myocarditis, valve stenosis, cardiac tamponade, aortic dissection, pulmonary embolism, high output syndrome or evidence of non-cardiovascular factors as main cause of symptoms. In order to assess the clinical significance of WRF only when caused by acute HF and/or its treatment, we also excluded patients who developed complications or underwent procedures which may cause a rise in $\mathrm{s}-\mathrm{Cr}$ during the hospitalisation. Namely, we excluded patients who had stroke, infection, shock, cardiac arrest, cardiac death or who underwent cardiac surgery or invasive procedures requiring contrast administration during the hospitalisation. We also excluded patients requiring dialysis or ultrafiltration, conditions with a clear prognostic impact in which tubular necrosis and organic renal damage are often present. Baseline s-Cr was not an exclusion criterion.

\subsection{Investigations}

All patients underwent a complete clinical and laboratory examination at the time of hospital admission, as well as serial laboratory measurements at 1 to 2 day intervals, and at hospital discharge. At least one Doppler-echocardiography exam was performed in all patients 1-3 days before discharge. Estimated glomerular filtration rate (GFR) was calculated by the Modification of Diet in Renal Disease (MDRD) equation. This has been shown to be the best method for the indirect assessment of renal function in HF patients [20,21]. Anaemia was defined according to the criteria of the World Health Organisation, when haemoglobin $(\mathrm{Hb})$ was $<13 \mathrm{~g} / \mathrm{dL}$ in men and $<12 \mathrm{~g} / \mathrm{dL}$ in women [22]. Furosemide doses were determined for: 1) the oral daily dose prior to hospital admission; 2) the total dose administered intravenously during the first day of hospitalisation; and 3) the oral daily dose administered at discharge. Thiazide diuretics (namely metolazone), dopamine and inotropic drugs (namely dobutamine or enoximone or levosimendan), were administered based on clinical indications as judged by the attending physician. This often occurred when insufficient diuresis was obtained after furosemide alone, in the case of thiazide diuretics, and when signs of peripheral hypoperfusion and/or WRF developed, in the case of dopamine and/or inotropic agents. Dopamine was always administered at low doses, as a 2 to $3 \mu \mathrm{g} / \mathrm{kg} / \mathrm{min}$ i.v. infusion.

Data collection and end-point adjudication was performed by independent investigators (GV, CL) who had no role in patient follow-up and treatment. Follow-up was performed by periodic (every 3 months) clinical visits and/or telephone calls to the patient or to her/his physician and her/his relatives. Relatives were instructed to inform the investigators as soon as possible, in case of any major cardiovascular events. Only cardiac death and urgent, unplanned hospitalisations were included as end-points of the study.

\subsection{Definition of $W R F$}

Worsening renal function was defined based on the maximal increase in $\mathrm{s}-\mathrm{Cr}$ from admission to any time during hospitalisation. The most widely used definition of WRF in previous studies has been an increase of $\geq 0.3 \mathrm{mg} / \mathrm{dL}$ in s-Cr $[8,10,13,15-17,23]$. However, the inverse relationship between glomerular filtration rate (GFR) and $\mathrm{s}-\mathrm{Cr}$ is exponential, so that small changes in s-Cr are attended by greater reductions in GFR at low initial s-Cr, compared to higher s-Cr levels [18]. Hence, to correct for the role of baseline s-Cr, we defined WRF as both a $\geq 0.3 \mathrm{mg} / \mathrm{dL}$ and a $\geq 25 \%$ increase in s-Cr from admission (WRF-Abs- $\%$ ). Our definition of WRF was compared to that based only on an increase in s-Cr $\geq 0.3 \mathrm{mg} / \mathrm{dL}$ (WRF-Abs) or $\geq 25 \%$ (WRF-\%) from baseline.

\subsection{Statistical analyses}

The primary objectives of the study were two-fold: first, to assess the prognostic value of these definitions of WRF, with respect to the prediction of cardiac death or HF hospitalisations occurring after discharge, and second, to assess the variables associated with developing WRF. Assuming a one-year 50\% incidence of major cardiovascular events (cardiovascular death or HF hospitalisations), we calculated that a sample size of 320 patients would provide 
$80 \%$ power, with an alpha value of 0.05 , to detect a $25 \%$ change in the risk of these events in the patients with WRF compared to those without WRF.

Continuous variables were expressed as mean \pm standard deviation, unless otherwise specified. Categorical variables were presented as percentage and compared by Yates corrected chi-square test. A two-tailed $p$ value $<0.05$ was considered significant. Comparisons between groups were performed by $t$-test or Wilcoxon test, as appropriate.

Independent predictors of WRF were identified by multivariable logistic regression analysis with backward stepwise regression amongst variables assessed prior to hospitalisation and on admission. All the variables which were different between patients with and without WRF at univariable analysis were entered into the initial model. The only exception was s-Cr on admission which was not entered in the model for the prediction of WRF, since it was included in the calculation of the percent change in s-Cr. Variables were entered at an entry level of significance of $p<0.1$ and kept in the model at an exit level of significance of $p<0.05$. For descriptive purposes, the analysis was repeated with continuous variables selected by the multivariable model dichotomised at their median value.

The combined end-point of cardiovascular mortality or unplanned HF hospitalisation was used as the primary outcome end-point. Cumulative event-free survival estimates were calculated using the Kaplan-Meier method. Patients were censored at the time of transplantation or of any cardiac surgical procedure. Differences in survival related to recognized major risk factors in HF patients [19] were evaluated by the Log-rank test. Variables which were different $(p<0.1)$ between patients with and without events were entered in a multivariable Cox regression model. The variables assessed were age, gender, body mass index, systolic blood pressure, heart rate, NYHA class, serum haemoglobin, creatinine, BUN, and sodium, both on admission and at discharge, left ventricular (LV) ejection fraction (EF) and a restrictive pattern of LV filling at Doppler-echocardiography, treatment with intravenous vasodilator and/or inotropic agents during the hospitalisation, prescription of angiotensin-converting enzyme inhibitors, angiotensin receptor blockers, beta-blockers, aldosterone antagonists, digoxin and/or furosemide at discharge, as well as daily furosemide doses during the hospitalisation and at discharge. Due to the similarities between the 3 definitions of WRF and the need to assess their prognostic value, we repeated the multivariable analysis entering each of the three definitions of WRF separately.

\section{Results}

\subsection{Patient characteristics}

Patient characteristics are listed in Table 1. The mean age was $68 \pm 11$ years and $60 \%$ were men. A relatively high percentage of co-morbid conditions were present, including
$34 \%$ of patients with diabetes, $24 \%$ with chronic renal failure, and $42 \%$ with anaemia. Left ventricular EF was $34 \pm$ $15 \%$, with 92 patients $(29 \%)$ having a LVEF $>45 \%$. All the patients were in New York Heart Association (NYHA) class III or IV at admission ( $51 \%$, in class III and $49 \%$, in class IV). The initial systolic blood pressure was $129 \pm 27 \mathrm{~mm} \mathrm{Hg}$ and heart rate $80 \pm 20 \mathrm{bpm}$. Pulmonary rales above the bases, peripheral oedema, signs of increased jugular venous pressure and hepatomegaly were present in $53 \%, 61 \%$, $31 \%$, and $40 \%$ of the patients, respectively.

Serum creatinine on admission was $1.51 \pm 0.84 \mathrm{mg} / \mathrm{dL}$ (range, 0.40 to $9.70 \mathrm{mg} / \mathrm{dL}$; median, interquartile range, [IQR] $1.30,1.00-1.70 \mathrm{mg} / \mathrm{dL}$ ); eGFR was $58 \pm 29 \mathrm{~mL} / \mathrm{min}$ (range, 6 to $234 \mathrm{~mL} / \mathrm{min}$; median, IQR, 56,33 to $62 \mathrm{~mL} / \mathrm{min}$ ). Worsening renal function, defined as a s-Cr increase of both $\geq 0.3 \mathrm{mg} / \mathrm{dL}$ and $\geq 25 \%$ from initial values (WRF-Abs-\%) was found in 107/318 patients (32\%) patients, versus 134/318 $(42 \%)$ and $110 / 318(35 \%)$ patients when only an increase $\geq 0.3 \mathrm{mg} / \mathrm{dL}$ and an increase $\geq 25 \%$ from baseline, were considered, respectively.

Table 2 lists the medical treatments of particular interest. More than $90 \%$ of the patients were on furosemide treatment, both before and after discharge. Seventy-eight percent of the patients were receiving an inhibitor of the renin-angiotensin system on entry with no changes at discharge, while there was an increase in the percentage of patients receiving a betablocker (from $57 \%$ to $77 \%$ ) and an aldosterone antagonist (from $45 \%$ to $55 \%$ ). With respect to in-hospital treatment, all patients received i.v. furosemide, 61 patients $(21 \%)$ received i.v. vasodilators (nitrates in 59, 19\%, and nitroprusside in 21 , $7 \%$ ) and $29(9 \%)$ received inotropic agents (dobutamine and/ or enoximone and/or levosimendan in 18, $6 \%, 13,4 \%$, and 5 , $2 \%$, patients, respectively).

After the initial hospitalisation, patients were followed for $480 \pm 363$ days (median, 388). During follow-up, 53 patients (17\%) died and $132(41 \%)$ were hospitalised for HF. Thirteen additional rehospitalisations occurred for other cardiovascular reasons (acute coronary syndrome, atrial fibrillation or stroke) with no signs of $\mathrm{HF}$ and were not considered as end-points. Eleven patients (3.4\%) were lost to follow-up and were excluded from the analysis.

\subsection{Characteristics of the patients with WRF}

The characteristics of the patients with and without WRFAbs- $\%$ are compared in Tables 1 and 2. The patients who developed WRF-Abs- $\%$ were more likely to be men and to have a history of pre-existing renal dysfunction. They also had more severe HF, as indicated by their more severe symptoms and signs of HF on admission, and had more severe impairment of LV systolic function by Dopplerechocardiography.

Prior to hospital admission, patients who developed WRF-Abs- $\%$ were receiving higher doses of furosemide and were less likely to be on beta-blockers, consistent with their more advanced HF. During the hospitalisation, patients who 
Table 1

Patients characteristics

\begin{tabular}{|c|c|c|c|c|}
\hline & Total & $\begin{array}{l}\text { No WRF- } \\
\text { Abs- } \%\end{array}$ & $\begin{array}{l}\text { WRF- } \\
\text { Abs- } \%\end{array}$ & $p$ value \\
\hline & $(n=318)$ & $(n=211)$ & $(n=107)$ & \\
\hline \multicolumn{5}{|l|}{ Demographics } \\
\hline Age, years & $68 \pm 11$ & $67 \pm 12$ & $69 \pm 9$ & 0.107 \\
\hline Male, $n(\%)$ & $190(60)$ & $117(55)$ & $73(68)$ & 0.038 \\
\hline \multicolumn{5}{|l|}{ Cause of HF, $n(\%)$} \\
\hline $\begin{array}{l}\text { Coronary artery } \\
\text { disease }\end{array}$ & $173(54)$ & $108(51)$ & $65(61)$ & \\
\hline $\begin{array}{l}\text { Idiopathic } \\
\text { cardiomyopathy }\end{array}$ & $120(38)$ & $84(40)$ & $36(34)$ & 0.459 \\
\hline Hypertension & $17(6)$ & $14(7)$ & $3(3)$ & \\
\hline Valvular heart disease & $8(2)$ & $5(2)$ & $3(3)$ & \\
\hline \multicolumn{5}{|l|}{ Body weight, kg } \\
\hline Entry & $77 \pm 15$ & $78 \pm 16$ & $76 \pm 15$ & 0.266 \\
\hline Discharge & $75 \pm 15$ & $75 \pm 15$ & $74 \pm 15$ & 0.275 \\
\hline$\Delta$, entry to discharge & $\begin{array}{l}-2.22 \pm \\
2.84\end{array}$ & $\begin{array}{l}-2.26 \pm \\
2.84\end{array}$ & $\begin{array}{l}-2.13 \pm \\
2.84\end{array}$ & 0.714 \\
\hline $\begin{array}{l}\text { Length of hospital } \\
\text { stay, days }\end{array}$ & $13 \pm 11$ & $10 \pm 8$ & $17 \pm 14$ & $<0.0001$ \\
\hline \multicolumn{5}{|l|}{ Medical history } \\
\hline Prior heart failure, $n, \%$ & $185(58)$ & $118(56)$ & $67(63)$ & 0.306 \\
\hline Hypertension, $n, \%$ & $170(53)$ & $106(50)$ & $64(60)$ & 0.134 \\
\hline Previous MI, $n, \%$ & $162(51)$ & $101(48)$ & $61(57)$ & 0.155 \\
\hline Diabetes, $n, \%$ & $91(29)$ & $59(30)$ & $32(30)$ & 0.790 \\
\hline Prior renal failure & $78(25)$ & $40(19)$ & $38(36)$ & 0.002 \\
\hline $\mathrm{COPD}, n, \%$ & 45 (14) & $25(12)$ & $20(19)$ & 0.138 \\
\hline Stroke, $n, \%$ & $22(7)$ & $13(6)$ & $9(8)$ & 0.608 \\
\hline PVD, $n, \%$ & $36(11)$ & $24(11)$ & $12(11)$ & 1 \\
\hline \multicolumn{5}{|l|}{ Electrocardiogram } \\
\hline QRS duration, ms & $130 \pm 40$ & $127 \pm 39$ & $136 \pm 41$ & 0.054 \\
\hline Atrial fibrillation, $n, \%$ & $98(31)$ & $57(27)$ & $41(38)$ & 0.053 \\
\hline $\mathrm{ICD}, n, \%$ & $89(28)$ & $52(25)$ & $37(35)$ & 0.083 \\
\hline Paced rhythm, $n, \%$ & $57(18)$ & $29(14)$ & $28(26)$ & 0.010 \\
\hline \multicolumn{2}{|l|}{ NYHA class } & & & \\
\hline Entry & $\begin{array}{l}3.48 \pm \\
0.50\end{array}$ & $\begin{array}{l}3.41 \pm \\
0.49\end{array}$ & $\begin{array}{l}3.62 \pm \\
0.49\end{array}$ & $<0.0001$ \\
\hline Discharge & $\begin{array}{l}2.04 \pm \\
0.74\end{array}$ & $\begin{array}{l}1.95 \pm \\
0.67\end{array}$ & $\begin{array}{l}2.22 \pm \\
0.85\end{array}$ & 0.002 \\
\hline \multicolumn{5}{|l|}{ Systolic BP, mm Hg } \\
\hline Entry & $129 \pm 27$ & $128 \pm 25$ & $131 \pm 29$ & 0.316 \\
\hline Discharge & $115 \pm 18$ & $116 \pm 18$ & $115 \pm 19$ & 0.557 \\
\hline \multicolumn{5}{|l|}{ Diastolic BP, mm Hg } \\
\hline Entry & $80 \pm 15$ & $79 \pm 15$ & $80 \pm 16$ & 0.644 \\
\hline Discharge & $72 \pm 10$ & $72 \pm 10$ & $71 \pm 10$ & 0.264 \\
\hline \multicolumn{5}{|l|}{ Heart rate, bpm } \\
\hline Entry & $80 \pm 20$ & $79 \pm 20$ & $83 \pm 20$ & 0.095 \\
\hline Discharge & $69 \pm 11$ & $68 \pm 10$ & $70 \pm 11$ & 0.169 \\
\hline \multicolumn{5}{|c|}{$\begin{array}{l}\text { Pulmonary rales or } \\
\text { crackles }>\text { basilar, } n(\%)\end{array}$} \\
\hline Entry & $170(53)$ & $98(46)$ & $72(67)$ & 0.001 \\
\hline Discharge & $20(6)$ & $10(5)$ & $10(9)$ & 0.176 \\
\hline \multicolumn{5}{|l|}{ Peripheral oedema, $n(\%)$} \\
\hline Entry & $107(34)$ & $58(27)$ & $49(46)$ & 0.002 \\
\hline Discharge & $6(2)$ & $2(1)$ & $4(4)$ & 0.196 \\
\hline \multicolumn{5}{|l|}{$\begin{array}{l}\text { Increased jugular venous } \\
\text { pressure, } n(\%)\end{array}$} \\
\hline Entry & $99(31)$ & $55(26)$ & $44(41)$ & 0.009 \\
\hline Discharge & $6(2)$ & $4(2)$ & $2(2)$ & 1.00 \\
\hline \multicolumn{5}{|l|}{ Hepatomegaly, $n(\%)$} \\
\hline Entry & $126(40)$ & $82(39)$ & $44(41)$ & 0.789 \\
\hline Discharge & $19(6)$ & $11(5)$ & $8(7)$ & 0.579 \\
\hline
\end{tabular}

Table 1 (continued)

\begin{tabular}{|c|c|c|c|c|}
\hline & Total & $\begin{array}{l}\text { No WRF- } \\
\text { Abs- } \%\end{array}$ & $\begin{array}{l}\text { WRF- } \\
\text { Abs- } \%\end{array}$ & $p$ value \\
\hline & $(n=318)$ & $(n=211)$ & $(n=107)$ & \\
\hline \multicolumn{5}{|l|}{ Laboratory exams } \\
\hline \multicolumn{5}{|l|}{ Haemoglobin, Gm/dL } \\
\hline Entry & $13.0 \pm 2.0$ & $13.1 \pm 2.1$ & $12.9 \pm 1.8$ & 0.244 \\
\hline Discharge & $12.7 \pm 1.94$ & $12.9 \pm 2.1$ & $12.4 \pm 1.7$ & 0.207 \\
\hline \multicolumn{5}{|l|}{ Anaemia, $n(\%)$} \\
\hline Entry & $134(42)$ & $81(38)$ & $53(50)$ & 0.007 \\
\hline Discharge & $170(53)$ & $104(49)$ & $66(62)$ & 0.048 \\
\hline \multicolumn{5}{|l|}{ Creatinine, $\mathrm{mg} / \mathrm{dL}$} \\
\hline Entry & $\begin{array}{l}1.51 \pm \\
0.84\end{array}$ & $\begin{array}{l}1.54 \pm \\
0.91\end{array}$ & $\begin{array}{l}1.45 \pm \\
0.67\end{array}$ & 0.360 \\
\hline Peak & $\begin{array}{l}1.82 \pm \\
1.05\end{array}$ & $\begin{array}{l}1.59 \pm \\
0.98\end{array}$ & $\begin{array}{l}2.26 \pm \\
1.06\end{array}$ & $<0.0001$ \\
\hline Discharge & $\begin{array}{l}1.55 \pm \\
0.76\end{array}$ & $\begin{array}{l}1.39 \pm \\
0.66\end{array}$ & $\begin{array}{l}1.88 \pm \\
0.84\end{array}$ & $<0.0001$ \\
\hline $\begin{array}{l}\Delta \text { entry to peak, } \\
\mathrm{mg} / \mathrm{dL}\end{array}$ & $\begin{array}{l}0.31 \pm \\
0.54\end{array}$ & $\begin{array}{l}0.05 \pm \\
0.20\end{array}$ & $\begin{array}{l}0.81 \pm \\
0.62\end{array}$ & $<0.0001$ \\
\hline$\Delta$ entry to peak, $\%$ & $11 \pm 23$ & $3 \pm 12$ & $62 \pm 52$ & $<0.0001$ \\
\hline \multicolumn{5}{|l|}{$\mathrm{BUN}, \mathrm{mg} / \mathrm{dL}$} \\
\hline Entry & $67 \pm 42$ & $66 \pm 44$ & $69 \pm 37$ & 0.592 \\
\hline Peak & $83 \pm 53$ & $74 \pm 45$ & $102 \pm 61$ & $<0.0001$ \\
\hline Discharge & $74 \pm 44$ & $68 \pm 39$ & $89 \pm 49$ & $<0.0001$ \\
\hline \multicolumn{5}{|l|}{$\mathrm{GFR}, \mathrm{mL} / \mathrm{min}$} \\
\hline Entry & $58 \pm 29$ & $56 \pm 24$ & $62 \pm 37$ & 0.105 \\
\hline Nadir & $49 \pm 24$ & $55 \pm 25$ & $36 \pm 10$ & $<0.0001$ \\
\hline Discharge & $56 \pm 26$ & $62 \pm 28$ & $45 \pm 20$ & $<0.0001$ \\
\hline \multicolumn{5}{|l|}{ Sodium, mEq/L } \\
\hline Entry & $139 \pm 4$ & $138 \pm 4$ & $139 \pm 4$ & 0.450 \\
\hline Discharge & $139 \pm 4$ & $139 \pm 4$ & $139 \pm 4$ & 0.826 \\
\hline \multicolumn{5}{|l|}{ Potassium, mEq/L } \\
\hline Entry & $4.1 \pm 0.5$ & $4.1 \pm 0.6$ & $4.1 \pm 0.5$ & 0.133 \\
\hline Discharge & $4.2 \pm 0.5$ & $4.2 \pm 0.5$ & $4.3 \pm 0.4$ & 0.102 \\
\hline Uric acid, mg/dL & $7.1 \pm 2.4$ & $6.9 \pm 2.2$ & $7.5 \pm 2.7$ & 0.074 \\
\hline Cholesterol,mg/dL & $177 \pm 44$ & $178 \pm 44$ & $174 \pm 43$ & 0.398 \\
\hline $\begin{array}{l}\text { Troponin I elevation, } \\
n(\%)\end{array}$ & $81(25)$ & $49(23)$ & $32(30)$ & 0.248 \\
\hline \multicolumn{5}{|l|}{ Doppler-echocardiography } \\
\hline LV Ejection fraction, \% & $\begin{array}{l}34.5 \pm \\
14.6\end{array}$ & $\begin{array}{l}36.0 \pm \\
15.0\end{array}$ & $\begin{array}{l}31.4 \pm \\
13.2\end{array}$ & 0.007 \\
\hline $\begin{array}{l}\text { Ejection Fraction }<45 \% \text {, } \\
\quad n, \%\end{array}$ & $226(71)$ & $141(67)$ & $85(79)$ & 0.027 \\
\hline LV dilation, $\%$ & $223(70)$ & $138(65)$ & 85 (79) & 0.001 \\
\hline Systolic PAPs, mm Hg & $44 \pm 12$ & $43 \pm 11$ & $47 \pm 13$ & 0.004 \\
\hline $\begin{array}{l}\text { Restrictive LV filling } \\
\text { pattern, } n, \%\end{array}$ & $129(41)$ & $75(35)$ & $54(50)$ & 0.015 \\
\hline Mitral regurgitation, $n, \%$ & $89(28)$ & $52(25)$ & $37(35)$ & 0.083 \\
\hline $\begin{array}{l}\text { Inferior vena cava } \\
\text { congestion, } n, \%\end{array}$ & $113(36)$ & $68(32)$ & $45(42)$ & 0.108 \\
\hline
\end{tabular}

Abbreviations: ICD, implantable defibrillator; MI, myocardial infarction; LV, left ventricular; MR, mitral regurgitation; PAP, pulmonary artery pressure; PVD, peripheral vascular disease; WRF, worsening renal function. Peak refers to the measurements performed at the time of peak serum creatinine levels.

developed WRF-Abs-\% received higher doses of both i.v. furosemide at entry and oral furosemide at discharge, and were more likely to be treated with i.v. dopamine and/or inotropic agents.

Using a multivariable model, including baseline clinical characteristics, co-morbidities, laboratory tests, and concomitant treatment, the only independent predictors of 
Table 2

Medical treatment

\begin{tabular}{|c|c|c|c|c|}
\hline & Total & No WRF-Abs- $\%$ & WRF-Abs- $\%$ & $p$ value \\
\hline & $(n=318)$ & $(n=211)$ & $(n=107)$ & \\
\hline Furosemide, $n,(\%)$ & & & & \\
\hline Entry & 315 (99) & 208 (99) & $107(100)$ & \\
\hline Dose, mg/day & $108 \pm 149$ & $82 \pm 122$ & $160 \pm 182$ & 0.532 \\
\hline median (IQR) & $50,25-125$ & $25,25-75$ & $50,25-250$ & 0.101 \\
\hline Discharge & $290(91)$ & $188(89)$ & $102(91)$ & \\
\hline Dose, mg/day & $100 \pm 116$ & $87 \pm 111$ & $126 \pm 122$ & $<0.001$ \\
\hline median (IQR) & $50,25-150$ & $50,25-125$ & $125,50-250$ & 0.005 \\
\hline $\mathrm{ACEi}$ and/or ARBs, & $n,(\%)$ & & & \\
\hline Entry & $248(78)$ & $165(78)$ & $83(78)$ & 1 \\
\hline Discharge & $245(77)$ & $164(78)$ & $81(76)$ & 0.791 \\
\hline Aldosterone antagor & ists, $n,(\%)$ & & & \\
\hline Entry & $143(45)$ & $94(46)$ & $49(45)$ & 0.927 \\
\hline Discharge & $185(55)$ & $117(64)$ & $68(58)$ & 0.206 \\
\hline Beta-blockers, $n,(\%$ & & & & \\
\hline Entry & $181(57)$ & $130(62)$ & $51(48)$ & 0.024 \\
\hline Discharge & $246(77)$ & $163(77)$ & $83(78)$ & 0.364 \\
\hline Digoxin, $n,(\%)$ & & & & \\
\hline Entry & $98(31)$ & $64(30)$ & $34(32)$ & 0.893 \\
\hline Discharge & $99(31)$ & $61(29)$ & $38(36)$ & 0.283 \\
\hline Thiazides, $n,(\%)$ & & & & \\
\hline Entry & $10(3)$ & $6(3)$ & $4(4)$ & 0.927 \\
\hline Discharge & $9(3)$ & $6(3)$ & $3(3)$ & 1 \\
\hline Amlodipine, $n,(\%)$ & & & & \\
\hline Entry & $20(6)$ & $10(5)$ & $10(9)$ & 0.176 \\
\hline Discharge & $19(6)$ & $13(6)$ & $6(6)$ & 1 \\
\hline ASA, $n,(\%)$ & & & & \\
\hline Entry & $106(33)$ & 79 (37) & $27(25)$ & 0.040 \\
\hline Discharge & $98(31)$ & $72(34)$ & $26(24)$ & 0.096 \\
\hline Ticlopidine/clopidog & rel, $n,(\%)$ & & & \\
\hline Entry & $62(20)$ & $41(19)$ & $21(20)$ & 1 \\
\hline Discharge & $81(25)$ & $49(23)$ & $32(30)$ & 0.248 \\
\hline Warfarin, $n,(\%)$ & & & & \\
\hline Entry & $56(18)$ & $30(14)$ & $26(24)$ & 0.038 \\
\hline Discharge & $52(16)$ & $26(12)$ & $26(24)$ & 0.010 \\
\hline Statins, $n,(\%)$ & & & & \\
\hline Entry & $117(37)$ & $80(37)$ & $37(37)$ & 0.646 \\
\hline Discharge & $143(45)$ & $95(45)$ & $48(45)$ & ns \\
\hline In-hospital i.v. treatr & nent & & & \\
\hline $\begin{array}{l}\text { I.v. furosemide, } \\
\text { mg/day }\end{array}$ & $190 \pm 228$ & $142 \pm 180$ & $285 \pm 279$ & $<0.001$ \\
\hline $\begin{array}{l}\text { Entry, mg/day } \\
\text { median, IQR }\end{array}$ & $95,40-250$ & $50,25-157$ & $250,80-500$ & \\
\hline $\begin{array}{l}\text { Vasodilatators, } \\
n, \%\end{array}$ & $67(21)$ & $38(18)$ & $29(27)$ & 0.083 \\
\hline Dopamine, $n, \%$ & $70(22)$ & $37(17)$ & $33(31)$ & 0.010 \\
\hline Inotropes, $n, \%$ & $29(9)$ & $10(5)$ & $19(18)$ & $<0.001$ \\
\hline
\end{tabular}

developing WRF-Abs-\% were history of chronic kidney disease, furosemide daily dose on admission, NYHA class, and LVEF (Table 3). The predictive value of the model for WRF-Abs- $\%$ was, however, low, with a sensitivity of $49 \%$ and a specificity of $74 \%$ when furosemide dose and LVEF were entered as continuous variables and a sensitivity of $41 \%$ and a specificity of $70 \%$ when they were entered as categorical variables. Similar results were found when patients were subdivided on the basis of only an increase $\geq 0.3 \mathrm{mg} / \mathrm{dL}$ (WRF-Abs) or $\geq 25 \%$ (WRF-\%) in $\mathrm{s}-\mathrm{Cr}$ (data not shown).
Table 3

Baseline determinants of WRF-Abs- $\%$ at multivariable analysis

\begin{tabular}{lll}
\hline Predictors & Odds ratio (95\% CI) & $p$ value \\
\hline Analysis with LVEF and furosemide dose as continuous variables & \\
History of chronic kidney disease & $3.66(1.61-8.33)$ & 0.002 \\
LV ejection fraction & $0.97(0.95-0.99)$ & 0.012 \\
I.v. furosemide daily dose on admission & $1.001(1.000-1.003)$ & 0.034 \\
NYHA class & $1.79(0.99-1.79)$ & 0.052 \\
& & \\
Analysis with LVEF and furosemide dose as categorical variables & \\
History of chronic kidney disease & $1.84(1.04-3.27)$ & $<0.0001$ \\
I.v. furosemide dose $>100 \mathrm{mg}$ /day & $2.18(1.27-3.73)$ & 0.004 \\
NYHA class (IV versus III) & $2.07(1.24-3.45)$ & 0.005 \\
LV ejection fraction $<30 \%$ & $1.66(1.01-2.75)$ & 0.047 \\
\hline
\end{tabular}

\subsection{Clinical and prognostic significance of WRF}

For the group as a whole, the mean duration of hospitalisation was $11 \pm 9$ days (median, 7 days; IQR, 6-15). Patients who developed WRF-Abs- $\%$ had a longer mean and median duration of hospital stay: $15 \pm 14$ days versus $8 \pm 7$ days (median, IQR: 12, 8-22 days versus 8, 5-14, days; $p<0.001$ ).

Patients who had developed WRF-Abs-\% during the index hospitalisation had a higher incidence of the combined end-point of subsequent cardiovascular death and $\mathrm{HF}$ hospitalisations (Fig. 1). Similar increases in the event rate were also found when patients were subdivided on the basis of WRF-\% and WRF-Abs. In a Cox proportional hazards multivariable analysis, WRF-Abs- $\%$ remained independently associated with cardiovascular mortality and $\mathrm{HF}$ hospitalisation (adjusted hazard ratio [HR], 1.47; 95\% confidence intervals $[\mathrm{CI}], 1.13-1.81 ; p=0.024)$. The other variables found to be significant in the model were peak

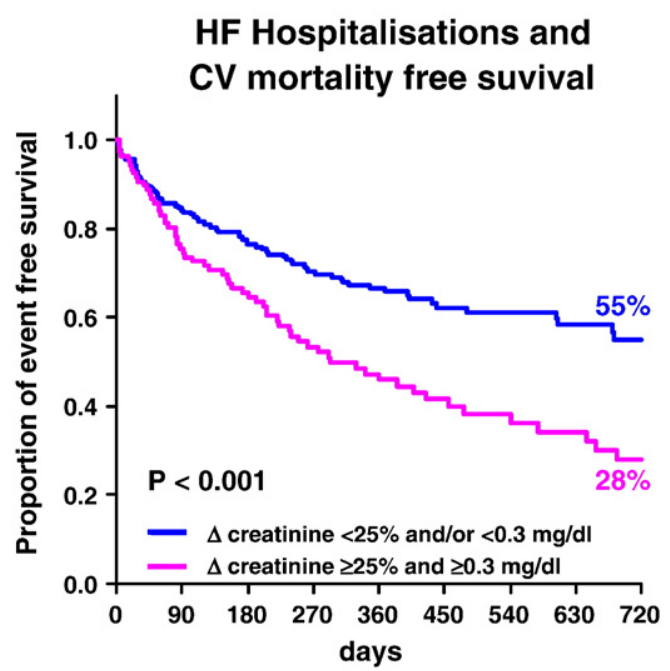

Pts. at risk

Absolute and percent s-Cr change:

$\begin{array}{llllll}<0.3 \text { or } 25 \% & 211 & 143 & 92 & 55 & 36 \\ \geq 0.3 \& 25 \% & 107 & 64 & 36 & 19 & 14\end{array}$

Fig. 1. Kaplan-Meier HF hospitalisations and cardiovascular mortality free survival curves for the patients subdivided on the basis of WRF development, defined as both $\geq 0.3 \mathrm{mg} / \mathrm{dL}$ and $\geq 25 \% \mathrm{~s}-\mathrm{Cr}$ increase from admission. 
furosemide dose administered during hospitalisation $(p<0.001)$, body weight decrease during hospitalisation $(p=0.001)$, systolic blood pressure at discharge $(p=0.002)$, diabetes $(p=0.012)$, a restrictive pattern of LV filling at predischarge Doppler-echocardiography ( $p=0.012)$, and serum haemoglobin levels $(p=0.037)$. Similar results were obtained with WRF-\%. In contrast, when WRF was defined only on the basis of an increase in $\mathrm{s}-\mathrm{Cr} \geq 0.3 \mathrm{mg} / \mathrm{dL}$, it was no longer a statistically significant independent predictor of outcome in the multivariable analysis. The other variables remained significant predictors, with the addition of BUN at discharge $(p=0.076)$.

Subgroup analysis showed that WRF-Abs-\% was associated with an increased event rate in all the subgroups without significant interactions based on either baseline clinical characteristics, parameters of LV systolic function or concomitant treatment.

\section{Discussion}

\subsection{Patient characteristics}

Our study confirms previous reports of the high prevalence of WRF in patients hospitalised for acute HF and its important impact on hospital length of stay and prognosis [9-11,13-17]. Our study differs from most of the previous studies, however, in that this was a prospectively designed single center protocol. Our patients had the characteristics of patients treated by a tertiary care HF center, i.e. they tended to be slightly younger, were less likely to be women, had a higher prevalence of previous HF and a lower LVEF, compared to the patients enrolled in some [10-12] but not other [13-17] previous larger studies, several of which included only patients $\geq 65$ years. The prevalence of concomitant diseases and, namely, of previous chronic renal failure as well as the s-Cr, BUN and eGFR values on admission of our patients were similar to those in previous studies $[5,9-17]$.

\subsection{Prevalence of $W R F$}

We used a different definition of WRF, which included not only the absolute increase in $\mathrm{s}-\mathrm{Cr}$ from values on admission but also required a $25 \%$ increase. This is important and, we believe, more appropriate since the same increase in s-Cr is, in fact, accompanied by a greater decline in GFR when it occurs from a low initial value [18]. Our definition, based on both absolute and percent $\mathrm{s}-\mathrm{Cr}$ changes, yielded a lower incidence of WRF (107/318 patients, $32 \%$ ) compared to the more traditional definition of WRF (i.e. an increase of s-Cr $\geq 0.3 \mathrm{mg} / \mathrm{dL}$ ), which was present in 134/318 patients (42\%) in our study. Similar results were found when WRF was defined based on a $\geq 25 \%$ increase in s-Cr. We believe that our study shows the greater clinical and prognostic value of WRF-Abs- $\%$ and WRF-\% compared to a definition based only on the absolute increase in s-Cr. The small size of our study group does not allow us to establish whether WRF-Abs-\% is better than WRF-\% only.

We found an incidence of WRF which was slightly higher than that reported in many $[9-11,14,15]$ but not all $[13,17]$ previous studies. This difference is likely caused by our protocol with frequent (every 1-2 days) reassessments of s$\mathrm{Cr}$ as well as by the higher prevalence of patients with advanced HF. Our study highlights the importance of frequent reassessments of renal function as the detection of WRF had prognostic significance.

\subsection{Causes of $W R F$}

The mechanisms which may cause WRF in patients with HF are multiple and are incompletely understood [6,24]. They include neurohormonal activation, decreased renal perfusion, and intrarenal mechanisms involving increased endothelin and/or adenosine release. Medical treatment may also have a significant role. In our study, daily intravenous furosemide dose, history of chronic kidney disease, NYHA class and LVEF were independent predictors of WRF.

Consistent with our data, the only other study in which the relationship of in-hospital medical treatment to subsequent WRF was assessed, also found that high doses of furosemide were associated with more frequent WRF [16]. Furosemide treatment, especially if at high doses, has been associated with a worse prognosis [25-28] and WRF may be a potential mechanism. On the other hand, we cannot exclude the possibility that the administration of higher doses of furosemide is a consequence, rather than a cause, of more advanced HF and coexistent renal failure. In this case, this would just be a marker, rather than a mechanism for poor outcomes. It is noteworthy that signs of congestion were more frequent in patients with WRF, whereas the actual decline in body weight was similar. This suggests that the patients had developed resistance to furosemide rather than having been subjected to excessive diuresis.

Diabetes [15-17], elevated systolic blood pressure $[10,15,16]$, a history of HF $[15,16]$ tachycardia and female gender [10] were all factors associated with increased risk of WRF in previous studies but this was not the case in our study. These differences are likely related to our smaller and more selected study group, such that some differences (e.g. history of HF) did not reach statistical significance and comorbidities were less important. Diabetes was, however, a significant determinant of subsequent mortality and hospitalisations.

\subsection{Clinical and prognostic value of WRF}

The length of hospital stay of our patients was comparable to that found in other European registries [29-32]. Our patients who developed WRF had a longer duration of hospital stay and this may be important both with respect to quality of life and the cost of treatment. Increased length of 
hospital stay is likely an effect of WRF as it causes changes in treatment aimed at improving renal function. Consistently, $\mathrm{s}-\mathrm{Cr}$ values at discharge were also lower than peak ones in our patients who developed WRF.

Worsening renal function was an independent predictor of subsequent mortality and HF hospitalisations. The other variables selected in our multivariable model were systolic blood pressure at discharge, body weight decrease during hospitalisation, diabetes, serum haemoglobin levels, peak furosemide dose during hospitalisation, and persistence of a restrictive pattern of LV filling at pre-discharge Dopplerechocardiography. These results are consistent with recent studies of prognostic variables in HF patients, both with regard to the variables selected and to the magnitude of their effect [27,32,33-36]. Renal function was significantly different in the patients with major cardiovascular events, compared to the others. However, when WRF-Abs- $\%$ was entered into our model, it lost its significance.

WRF-Abs has been shown to be associated with a poor prognosis in most [9-11,13-16] but not all [17] of the previous studies. Some of these studies, however, did not assess the prognostic value of WRF compared with other variables by multivariable analysis $[11,13,14]$. Our results are consistent with those of Cowie et al. who found that an increase in s-Cr by $0.3 \mathrm{mg} / \mathrm{dL}$ is associated with a poor prognosis in univariate, but not multivariable analysis [17]. WRF-Abs-\%, unlike WRF-Abs, remained significantly related to prognosis after adjustment for the other variables. This difference is likely related to the exponential relation between s-Cr and GFR so that WRF, expressed as both an absolute and a percentage change, also takes into account initial s-Cr values [18].

\subsection{Limitations of the study}

Our study is limited by the relatively low number of patients and events. It is likely that a larger study group with a greater number of events would have allowed the identification of an independent prognostic role of other variables such as, for instance, serum creatinine at discharge. However, as pointed out by the statistical power calculation, the size of our group was sufficient to detect a significant association between WRF and outcomes, which was the primary aim of our study.

Another limitation may be found in the possibility of biases during follow-up favouring rehospitalisations in the patients who had developed WRF during the initial hospitalisation and vice versa. However, only urgent, unplanned hospitalisations were included as end-points and these events have a low likelihood to be influenced by the investigator.

\section{Conclusions}

Our study demonstrates that WRF is a common finding in patients hospitalised for acute HF. Patients who developed WRF were more likely to have a history of chronic kidney disease, had more severe HF and were treated with higher doses of furosemide. When defined as both an absolute and a percent increase from baseline, WRF is an especially powerful and independent predictor of subsequent cardiovascular mortality and HF hospitalisations.

\section{References}

[1] Dries DL, Exner DV, Domanski MJ, Greenberg B, Stevenson LW. The prognostic implications of renal insufficiency in asymptomatic and symptomatic patients with left ventricular systolic dysfunction. J Am Coll Cardiol 2000;35:681-9.

[2] Hillege HL, Girbes AR, de Kam PJ, et al. Renal function, neurohormonal activation, and survival in patients with chronic heart failure. Circulation 2000;102:203-10.

[3] Mahon NG, Blackstone EH, Francis GS, Starling III RC, Young JB, Lauer MS. The prognostic value of estimated creatinine clearance alongside functional capacity in ambulatory patients with chronic congestive heart failure. J Am Coll Cardiol 2002;40(6):1106-13.

[4] Shlipak MG, Smith GL, Rathore SS, Massie BM, Krumholz HM. Renal function, digoxin therapy, and heart failure outcomes: evidence from the digoxin intervention group trial. J Am Soc Nephrol 2004; 15:2195-203.

[5] McAlister FA, Ezekowitz J, Tonelli M, Armstrong PW. Renal insufficiency and heart failure: prognostic and therapeutic implications from a prospective cohort study. Circulation 2004;109:1004-9.

[6] Shlipak MG, Massie BM. The clinical challenge of cardiorenal syndrome. Circulation 2004;110:1514-7.

[7] Smith GL, Lichtman JH, Bracken MB, et al. Renal impairment and outcomes in heart failure: systematic review and meta-analysis. J Am Coll Cardiol 2006;47:1987-96.

[8] de Silva R, Nikitin NP, Witte KK, et al. Incidence of renal dysfunction over 6 months in patients with chronic heart failure due to left ventricular systolic dysfunction: contributing factors and relationship to prognosis. Eur Heart J 2006;27:569-81.

[9] Akhter MW, Aronson D, Bitar F, et al. Effect of elevated admission serum creatinine and its worsening on outcome in hospitalized patients with decompensated heart failure. Am J Cardiol 2004;94:957-60.

[10] Krumholz HM, Chen YT, Vaccarino V, et al. Correlates and impact on outcomes of worsening renal function in patients $>$ or $=65$ years of age with heart failure. Am J Cardiol 2000;85:1110-3.

[11] Smith GL, Vaccarino V, Kosiborod M, et al. Worsening renal function: what is a clinically meaningful change in creatinine during hospitalization with heart failure? J Card Fail 2003;9:13-25.

[12] van Kimmenade RRJ, Januzzi Jr JL, Baggish AL, et al. Aminoterminal pro-brain natriuretic peptide, renal function, and outcomes in acute heart failure: redefining the cardiorenal interaction? J Am Coll Cardiol 2006;48:1621-7.

[13] Gottlieb SS, Abraham W, Butler J, et al. The prognostic importance of different definitions of worsening renal function in congestive heart failure. J Card Fail 2002;8:136-41.

[14] Weinfeld MS, Chertow GM, Stevenson LW. Aggravated renal dysfunction during intensive therapy for advanced chronic heart failure. Am Heart J 1999;138:285-90.

[15] Forman DE, Butler J, Wang Y, et al. Incidence, predictors at admission, and impact of worsening renal function among patients hospitalized with heart failure. J Am Coll Cardiol 2004;43:61-7.

[16] Butler J, Forman DE, Abraham WT, et al. Relationship between heart failure treatment and development of worsening renal function among hospitalized patients. Am Heart J 2004;147:331-8.

[17] Cowie MR, Komajda M, Murray-Thomas T, Underwood J, Ticho B. Prevalence and impact of worsening renal function in patients hospitalized with decompensated heart failure: results of the prospective outcomes study in heart failure (POSH). Eur Heart J 2006;27:1216-22. 
[18] Stevens LA, Coresh J, Greene T, Levey AS. Assessing kidney function-measured and estimated glomerular filtration rate. $\mathrm{N}$ Engl $\mathrm{J}$ Med 2006;354:2473-83.

[19] Nieminen MS, Bohm M, Cowie MR, et al. Executive summary of the guidelines on the diagnosis and treatment of acute heart failure: the Task Force on Acute Heart Failure of the European Society of Cardiology. Eur Heart J 2005;26:384-416.

[20] O'meara E, Chong KS, Gardner RS, Jardine AG, Neilly JB, McDonagh TA. The Modification of Diet in Renal Disease (MDRD) equations provide valid estimations of glomerular filtration rates in patients with advanced heart failure. Eur J Heart Fail 2006;8:63-7.

[21] Smilde TD, van Veldhuisen DJ, Navis G, Voors AA, Hillege HL. Drawbacks and prognostic value of formulas estimating renal function in patients with chronic heart failure and systolic dysfunction. Circulation 2006;114:1572-80.

[22] Izaks GJ, Westendorp RG, Knook DL. The definition of anemia in older persons. JAMA 1999;281:1714-7.

[23] Pitt B, Segal R, Martinez FA, Meurers G, et al. Randomised trial of losartan versus captopril in patients over 65 with heart failure (Evaluation of Losartan in the Elderly Study, ELITE). Lancet 1997;349:747-52.

[24] Lapman PG, Golduber GN, Le Jemtel TH. Heart failure treatment and renal function. Am Heart J 2004;147:193-4.

[25] Neuberg GW, Miller AB, O'Connor CM, et al. Diuretic resistance predicts mortality in patients with advanced heart failure. Am Heart $\mathrm{J}$ 2002;144:31-8.

[26] Eshaghian S, Horwich TB, Fonarow GC. Relation of loop diuretic dose to mortality in advanced heart failure. Am J Cardiol 2006;97:1759-64.

[27] Levy WC, Mozaffarian D, Linker DT, et al. The Seattle Heart Failure Model: prediction of survival in heart failure. Circulation 2006;113: $1424-33$.
[28] Hasselblad V, Gattis Stough W, Shah MR, et al. Relation between dose of loop diuretics and outcomes in a heart failure population: results of the ESCAPE trial. Eur J Heart Fail 2007;9:1064-9.

[29] Rudiger A, Harjola VP, Muller A, et al. Acute heart failure: clinical presentation, one-year mortality and prognostic factors. Eur J Heart Fail 2005;7:662-70.

[30] Zannad F, Mebazaa A, Juilliére Y, et al. Clinical profile, contemporary management and one-year mortality in patients with severe acute heart failure syndromes: the EFICA study. Eur J Heart Fail 2006;8:697-705.

[31] Nieminen MS, Brutsaert D, Dickstein K, et al. EuroHeart Failure Survey II (EHFS II): a survey on hospitalized acute heart failure patients: description of population. Eur Heart J 2006;27:2725-36.

[32] Tavazzi L, Maggioni AP, Lucci D, et al. Nationwide survey on acute heart failure in cardiology ward services in Italy. Eur Heart J 2006;27:1207-15.

[33] Aronson D, Mittleman MA, Burger AJ. Elevated blood urea nitrogen level as a predictor of mortality in patients admitted for decompensated heart failure. Am J Med 2004;116:466-73.

[34] Fonarow GC, Adams Jr KF, Abraham WT, Yancy CW, Boscardin WJ. ADHERE Scientific Advisory Committee, Study Group, and Investigators. risk stratification for in-hospital mortality in acutely decompensated heart failure: classification and regression tree analysis. JAMA 2005;293:572-80.

[35] Pocock SJ, Wang D, Pfeffer MA, et al. Predictors of mortality and morbidity in patients with chronic heart failure. Eur Heart J 2006;27:65-75.

[36] Gheorghiade M, Abraham WT, Albert NM, et al. Systolic blood pressure at admission, clinical characteristics, and outcomes in patients hospitalized with acute heart failure. JAMA 2006;296:2217-26. 